\title{
Mesenchymal stem cells as a treatment for neonatal ischemic brain damage
}

\author{
Cindy T.J. van Velthoven', Annemieke Kavelaars' and Cobi J. Heijnen'
}

Mesenchymal stem cell (MSC)-based therapies have been proven effective in experimental models of numerous disorders. Treatment of ischemic brain injury by transplantation of MSCs in neonatal animal models has been shown to be effective in reducing lesion volume and improving functional outcome. The beneficial effect of MSC transplantation to treat neonatal brain injury might be explained by the great plasticity of the neonatal brain. The neonatal brain is still in a developmentally active phase, leading to a better efficiency of MSC transplantation than that observed in experiments using adult models of stroke. Enhanced neurogenesis and axonal remodeling likely underlie the improved functional outcome following MSC treatment after neonatal hypoxic-ischemic (HI) brain injury. With respect to the mechanism of repair by MSCs, MSCs do not survive long term and replace damaged tissue themselves. We propose that MSCs react to the needs of the ischemic cerebral environment by secretion of several growth factors, cytokines, and other bioactive molecules to regulate damage and repair processes. Parenchymal cells react to the secretome of the MSCs and contribute to stimulate repair processes. These intrinsic adaptive properties of MSCs make them excellent candidates for a novel therapy to treat the devastating effects of $\mathrm{HI}$ encephalopathy in the human neonate.

$\mathbf{E}^{\mathrm{n}}$ ncephalopathy due to hypoxic-ischemic (HI) events occur when blood supply to the brain is interrupted, leading to diminished delivery of oxygen and nutrients to the tissue. The neonatal brain is particularly vulnerable for diminished blood flow because of its high energy need, which almost all comes in the form of glucose. When oxygen and glucose delivery become insufficient to meet the metabolic demands of the brain, a sequence of biochemical events will be triggered, including initial energy failure, calcium overload, excitotoxicity, oxidative stress, inflammation and apoptosis, autophagy, and necrosis, leading to global brain injury $(1,2)$.
Current therapeutic intervention strategies for infants with ischemic brain injury are limited and predominantly focused on the prevention of apoptosis, necrosis, and inflammation (neuroprotection) during the early phase of brain injury. For neonates born at term with $\mathrm{HI}$ brain injury, hypothermia (i.e., cooling of the brain by 2 to $4{ }^{\circ} \mathrm{C}$ ) has emerged as the only reliable intervention at present $(3,4)$.

Mesenchymal stem cells (MSCs) can also be neuroprotective because the cells have important anti-inflammatory and antiapoptotic properties (5-8). Administration of MSCs 3 to $24 \mathrm{~h}$ after stroke in adult animals reduced the number of apoptotic cells by approximately $50 \%$ in the ischemic boundary zone, which was accompanied by an increased expression of antiapoptotic proteins Bcl-2 and survivin (5). These results indicate that transplantation of MSCs in the very early phase after ischemic brain injury is neuroprotective and thereby improves functional outcome. However, an important drawback of neuroprotective strategies like hypothermia is the time window since treatment in the case of hypothermia must be initiated within $6 \mathrm{~h}$ after onset of the injury. This poses a problem for the neonatologist because the 6-h time window is often too short to be able to start therapy. However, once brain damage has become established, the resulting injured tissue is no longer amenable for neuroprotection. Like hypothermia, the great limitation with most neuroprotective strategies is that it must be applied within a couple of hours after onset of injury. Therefore, there is an urgent need for a therapy applicable when brain damage has already been established. The ultimate aim for an efficient therapy with an extended time window after ischemic brain injury should stimulate functional repair of brain tissue.

\section{MSC TRANSPLANTATION}

A variety of exogenous stem cells have been studied in experimental models for their potential to treat neonatal ischemic brain injury, including MSCs (9). MSCs can be easily harvested not only from bone marrow and adipose tissue but also from extraembryonic tissues such as placental tissue and Wharton's jelly, the stroma of the umbilical cord $(10,11)$. The latter sources are interesting because many neonates will experience an $\mathrm{HI}$ insult around the time of birth, at which moment 
the cells could be harvested from the placenta or the umbilical cord and transplanted as an autologous source. Oxygen tension is an important regulator of MSC function and mild hypoxia has been shown to stimulate MSC survival, proliferation, and stem cell potential $(12,13)$. This makes it plausible that MSCs derived from the hypoxic neonate could even have a better therapeutic potential.

As stated above, MSCs have important immunomodulatory properties, including a suppressive effect on $\mathrm{T}$ - and B-cell proliferation, suppression of natural killer cell function, and modulation of the secretory profile of dendritic cells and macrophages $(7,8)$. These properties might contribute to the low immunogenicity of MSCs after allogeneic transplantation. The anti-inflammatory and immunomodulatory MSCs could allow for allogeneic transplantation $(14,15)$. There is some evidence, however, that MSCs may have both cellular and humoral alloreactivity, such as activation of $\mathrm{T}$-cells that may reduce the capacity of allogeneic MSC transplantation (16,17). Yet MSC immunogenicity is still considerably attenuated compared with other allogeneic cell types because of their intrinsic antiinflammatory and immunomodulatory properties and absent to low major histocompatibility class II antigen expression $(16,18)$. In addition, the site of administration is an important factor in determining the immunogenicity of allogeneic MSCs because intracranially or intracerebrally administered allogeneic MSCs appear to be nonimmunogenic to weakly immunogenic $(19,20)$, whereas allogeneic MSCs administered intravenously or intraperitoneally sometimes elicit an antidonor response $(21,22)$. However, in some disease models, like myocardial infarction and stroke, allogeneic and autologous MSCs were found to be similarly effective in stimulating repair despite induction of an antigraft immune response (16). Although more preclinical research is needed, the use of an allogeneic source of MSCs would be a major advantage because it allows the availability of cells from healthy donors "off the shelf."

Transplantation of MSCs has been applied successfully in animal models for adult neurological disorders including cerebral ischemia, Alzheimer's disease, and Parkinson's disease (9,23-25). Several experimental studies in neonatal models have shown that treatment with MSCs after ischemic brain injury has beneficial effects on functional outcome (26-29). Lateralizing motor deficits are decreased by $40-70 \%$ after treatment with MSCs, although a decrease in lesion volume is not always reported (26-29).

Various routes of administration of MSCs have been applied in experimental models for transplantation to the brain. Intracranial injection of MSCs after neonatal ischemic brain injury in rodents improved functional recovery and reduced lesion size (27-29). Moreover, MSCs transplanted via the intravenous or intracardiac route also improved functional recovery $(26,30)$. To circumvent the invasive procedure of local intracranial administration and the loss of cells after peripheral administration, we studied the possibility of using the intranasal administration route (31). Upon intranasal administration, MSCs are capable of crossing the cribiform palate and migrating throughout the brain. Intranasal administration of MSCs after neonatal HI resulted in reduced lesion volume and improved motor function (32). Although a direct comparison between the regenerative efficiency of the intranasal and intracranial administration route cannot be made because a different number of cells was used, it is remarkable that the functional outcomes of the intranasal and intracranial route are very much alike (Figure 1).

In the study by Yasuhara et al. (30), a direct comparison was made between intracranial and intravenous administration of MSCs. Their results showed that even when using different

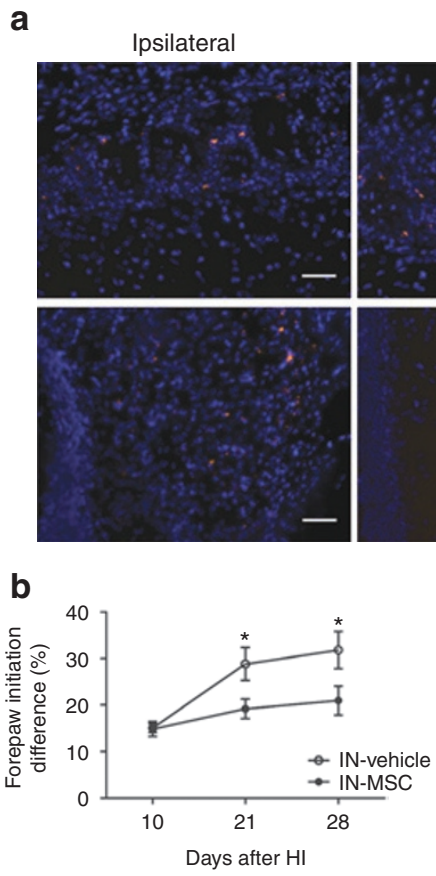

d
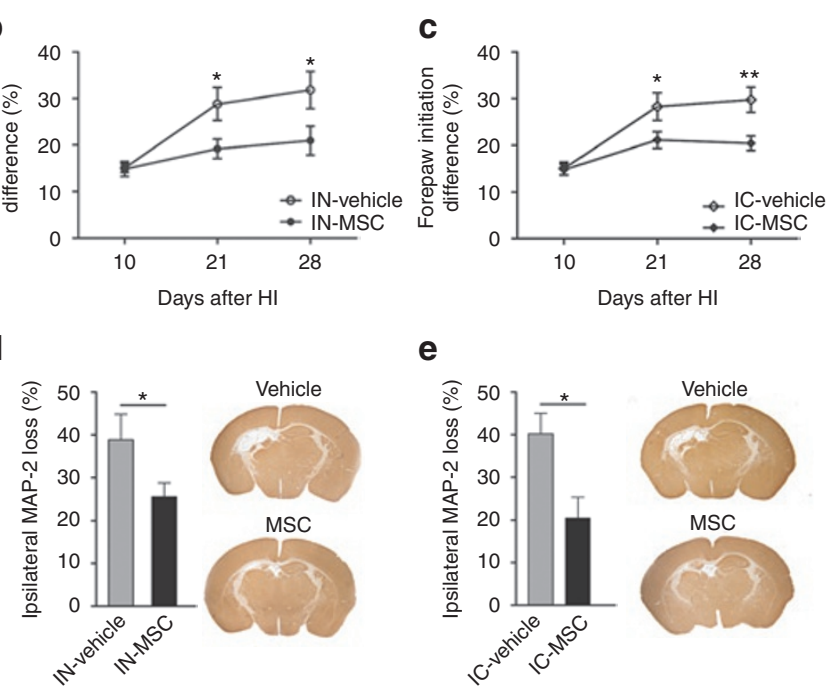

e

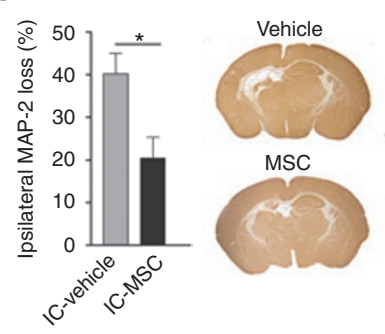

Figure 1. Effect of intranasal and intracranial mesenchymal stem cell (MSC) treatment following neonatal hypoxia-ischemia (HI). Brain sections $18 \mathrm{~d}$ after intranasal administration of MSCs at $10 \mathrm{~d}$ after $\mathrm{HI}$ (a). Sections were stained with 4',6-diamidino-2-phenylindole (blue) to visualize all nuclei. MSCs (red) were found in both the ipsi- and the contralateral olfactory bulb. In the contralateral hippocampus no MSCs could be found. However, in the damaged ipsilateral hippocampus, many MSCs are present. Scale bar represents $50 \mu \mathrm{m}$. Preference for use of the impaired paw was determined as a measure of sensorimotor function after intranasal (IN) MSC treatment (b) and intracranial (IC) MSC treatment (c). Quantification of lesion size after intranasal MSC treatment (d) and intracranial MSC treatment (e) was performed by measuring the ratio of ipsilateral to contralateral MAP2 ${ }^{+}$. Data represent means \pm SEM. For vehicle groups $n=8$, and for MSC groups $n=12$. $\left({ }^{*} P<0.05,{ }^{* *} P<0.01\right)$. (Reprinted with permission from C.T. van Velthoven, Pediatr Res 2010;68:419-22, Copyright @ 2010 International Pediatric Research Foundation.) 
administration routes and equal number of MSCs, the outcome of the regenerative response is equal. This indicates that the administration route of MSCs is not a key factor in determining the outcome in neonatal ischemic brain injury models. Although the results may be equal, the number of MSCs needed for systemic administration is much higher than the number of MSCs needed for local administration in the brain, because several studies showed that although intravenous administration of MSCs improved repair of brain injury, many intravenously administered MSCs are trapped in the lung (33). However, after peripheral administration, MSCs are able to migrate toward the lesion site (34). In vivo evidence showed that MSC homing toward the ischemic lesion site is regulated via interaction between stromal cell-derived factor-1 $1 \alpha$ and its receptor CXCR4 present on MSCs (35). In response to $\mathrm{HI}$ brain injury, stromal cell-derived factor-1a expression is upregulated near the lesion site and expressed for at least $14 \mathrm{~d}$ after induction of the injury (36). MSCs that express CXCR4 migrate to the lesioned area in the brain after intravenous transplantation, whereas MSCs derived from CXCR4 knockout mice do not migrate to the lesion (37). Furthermore, in response to in vitro culturing of MSCs with ischemic brain extract, MSCs increase the expression of CXCR4 on their membrane (32) and could thereby increase their migration toward the lesion site.

The initial studies on application of MSCs for ischemic brain injury focused on the capacity of MSCs to replace lost cells by migrating toward the lesion site and to differentiate into neurons $(38,39)$. More recently, however, this focus shifted toward the ability of MSC to exert their effect via stimulation of endogenous trophic mechanisms $(6,40,41)$. This shift in interest originates from the observation that long-term engraftment is not a necessity for MSCs to exert their regenerative function $(25,33,41)$. Previously, we have shown that MSC treatment decreases lesion volume after neonatal $\mathrm{HI}$, whereas $3 \mathrm{~d}$ after a single intracranial MSC injection, only $\sim 13 \%$ of transplanted cells could be detected in the brain $(28,29,41)$. Although the detected number of intracranial transplanted cells is twofold higher after double MSC treatment, the majority of cells do not survive up to $3 \mathrm{~d}$ after transplantation (41). After intranasal or intracardiac administration of MSCs, it was possible to detect the cells in the brain over longer periods of time up to $6 \mathrm{wk}$ after transplantation $(26,32)$. It could be argued that migration of MSCs to the lesion site allows for a better adaptation of MSCs to the harsh ischemic environment, leading to better MSC survival than after intracranial transplantation early after transplantation. However, the number of MSCs surviving up to $4 \mathrm{wk}$ after intracranial, intravenous, intracardiac, and intranasal transplantation is less than $1 \%(26,30,32,41)$, so the administration route has little to no influence on late graft survival. This number of surviving cells remains small in relation to the lesion volume and the number of MSCs that may integrate into the cerebral network is too little to cause the impressive effects just by mere differentiation of transplanted MSCs. Still, it cannot be completely excluded that some of the transplanted cells will differentiate and integrate into the network.
Another, perhaps more realistic, mechanism of action, therefore, is that transplanted MSCs improve neurological outcome by creating a growth-promoting niche via paracrine mechanisms. Thereby an environment is created in which endogenous repair processes and endogenous neuronal stem cells are induced to repair the damage.

\section{GROWTH-PROMOTING ENVIRONMENT}

MSCs secrete many factors that can influence important processes like apoptosis, neurogenesis, angiogenesis, and synaptogenesis $(6,29)$. The environment in the brain, defined as the capacity of resident cells to produce growth and differentiation factors, is a key factor in determining the response of MSCs after transplantation.

MSCs respond to the microenvironment in the brain and induce changes to that microenvironment (Figure 2) $(29,41)$. When MSCs are cultured in vitro with ischemic brain extracts, the cells respond to the ischemic extract by changing their expression of several secreted factors that are known to regulate cell proliferation, differentiation, and/or maturation in the brain $(41,42)$. In contrast to in vitro culture of MSCs with ischemic brain extracts, we observed that MSCs cultured with ischemic brain extracts from mice treated with MSCs at d 3 after HI express proinflammatory cytokines like interleukin (IL)-1 $\beta$ and IL-6 (29). Although inflammation is viewed as detrimental to neuroplasticity, a certain level of inflammation is probably needed for proper repair (43). Interestingly, both IL- $1 \beta$ and IL-6 promote neuron survival after excitotoxicity and IL- $1 \beta$ promotes oligodendrocyte remyelination after excitotoxicity, stimulates neurite outgrowth and nerve regeneration in neuronal cultures, and promotes Schwann cell proliferation (44-46). On the basis of our in vitro experiments, we conclude that MSCs

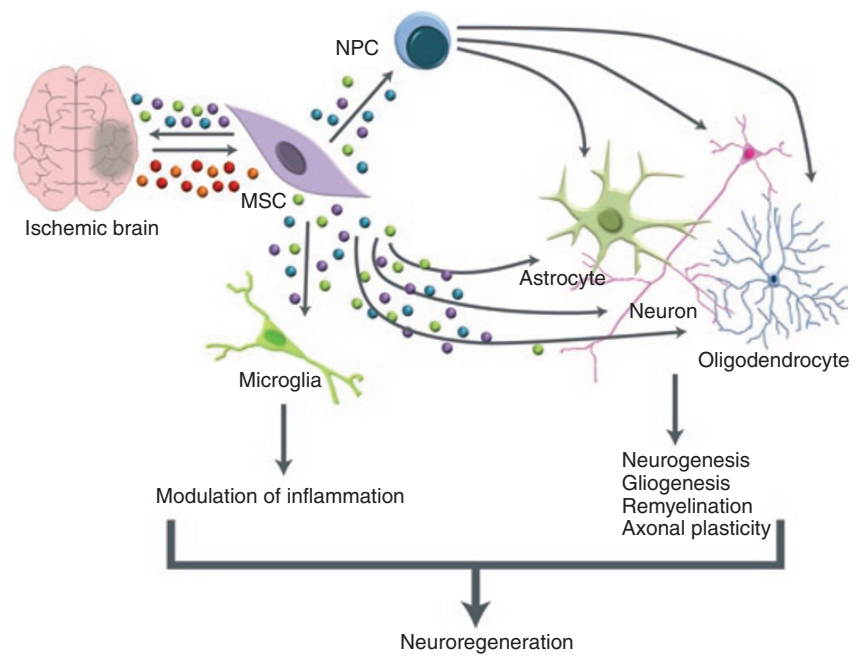

Figure 2. Mesenchymal stem cell (MSC)-induced neuroregeneration after neonatal hypoxia-ischemia ( $\mathrm{HI})$. After transplantation into the ischemic brain MSCs respond to the environment by producing growth and differentiation factors. These factors stimulate proliferation and differentiation of neural stem cells (NSCs) and stimulate repair processes. Upon transplantation of MSCs, microglia are activated and modulate the inflammatory response of the brain contributing to neurorepair. 
sense the microenvironment and change their expression profile according to the needs of this milieu.

We have shown before that repeated administration of MSCs at 3 and $10 \mathrm{~d}$ after neonatal $\mathrm{HI}$ markedly improves functional outcome, reduces infarct volume, and stimulates neurogenesis (29). More importantly, each injection of MSCs had distinct effects on regenerative processes. A single injection stimulates cell proliferation in the brain, whereas the second injection of MSCs on d 10 after HI stimulates axonal remodeling (29).

Gene expression profiling of the neonatal ischemic hemisphere, after a single administration of MSCs at $3 \mathrm{~d}$ after HI, showed that expression of fibroblast growth factor-2, epidermal growth factor, glial cell line-derived neurotrophic factor, and Sonic hedgehog is upregulated (41). These factors have multiple functions including a great potential to stimulate formation of new functional neurons in the lesioned area. Fibroblast growth factor-2 and epidermal growth factor are important in maintaining potency of neural stem cells and potent stimuli of progenitor cell proliferation in the neurogenic regions $(47,48)$. Administration of glial cell line-derived neurotrophic factor after adult stroke results in less cell death and glial cell linederived neurotrophic factor stimulates subventricular zone neurogenesis and migration of precursor cells toward the site where new neurons are needed $(49,50)$. Sonic hedgehog is a powerful factor that stimulates neural progenitor cell proliferation, migration, and differentiation toward neurons and oligodendrocytes and could thereby contribute to both gray and white matter remodeling (51).

Interestingly, a second transplantation with MSCs induces not only a gene expression profile similar to the changes seen after a single transplantation, but also expression of additional genes that tip the balance toward a milieu that favors end-stage differentiation and neuronal network development in the ischemic brain, such as expression of neuropilin-1 and 2, neuregulin-1, and EphrinB2 (41). These molecules are important in axon guidance and synapse formation, processes needed for functional integration of new neurons into the neuronal network (52).

The data obtained by gene expression analysis of the ischemic hemisphere after single or double MSC transplantation fit well with the in vivo data, showing that the distinct effects of MSC treatment on regenerative processes are related to changes in the growth factor environment in the brain $(29,41)$. A first administration of MSCs stimulates cell proliferation in the brain $(28,29)$. For these new cells to become functional neurons, they must differentiate into mature brain cells. This process will be driven by growth and differentiation factors provided by the milieu in the brain after MSC transplantation. However, it is unlikely that the factors secreted by the MSCs will be solely responsible for the extensive changes involved in endogenous neuroplasticity in the ischemic microenvironment. MSC treatment will stimulate parenchymal cells to secrete an array of trophic factors that will enhance neuroplasticity $(40,50)$. The signals responsible for guiding progenitor cells through the various repair processes such as neurogenesis, gliogenesis, axonal sprouting, and synaptogenesis are complex and depend on a balance between various intra- and extracellular molecules $(53,54)$. Manipulation of this balance by improving and directing the therapeutic potential of MSCs might reflect a promising future approach to better target regenerative therapy, although it may prove to be difficult. Genetic manipulation of MSCs may be one efficient way to direct the repair process as well as to increase the capacity of the endogenous repair of the brain (55).

\section{NEUROGENESIS AND REGENERATION OF THE BRAIN}

Following HI, there is an initial decrease in proliferation of neural progenitor cells (NPCs) and astrocytes in the ipsilateral dentate gyrus (28), when HI-induced cell death is still ongoing in the brain (2). After the initial decrease, cell proliferation starts to increase and peaks at $72 \mathrm{~h}$ after HI (41). The proliferating cells after neonatal HI express markers of immature multipotent precursors, but not markers of lineage-restricted progenitor cells (56), leaving the possibility of replacing different types of lost cells. In the healthy adult brain, neurogenesis is necessary to maintain neurological function. Ablation of NPCs from the dentate gyrus reduces neurogenesis and impairs functional plasticity, showing the importance and necessity of neurogenesis after brain injury $(57,58)$.

The number of NPCs is relatively high in the newborn brain. More importantly, parenchymal astrocytes in the neonatal brain may still have stem cell properties compared with the adult brain (59), suggesting that the neonatal brain has a higher capacity to heal itself after injury.

Transplantation of MSCs further stimulated HI-induced NPC proliferation in the lesioned hemisphere and enhanced survival of proliferating cells after migration toward the lesion site (28). A vast number of proliferating progenitor cells will ultimately differentiate into neurons, astrocytes, and oligodendrocytes (Figure 2) $(28,56)$. Astrocytes play an important role in the repair processes that take place after injury. In the healthy brain, astrocytes have many functions including provision of structural and metabolic support to neurons, regulation of the extracellular ionic environment, and elimination of excessive levels of neurotransmitters such as glutamate (60). After brain injury, astrocytes become "reactive," which involves proliferation, cellular hypertrophy, changes in gene expression, and release of growth-promoting and -inhibiting factors $(60,61)$. In the hippocampus, the most injured area after experimental neonatal HI, MSC treatment increased the number of dividing astrocytes, whereas in the cortex, an area that sustained less damage, the number of new astrocytes was decreased after MSC treatment (28). This indicates a relationship between damage and the local need for differentiation of newly divided cells toward astrocytes after MSC treatment. More proliferating astrocytes around the lesion site reflect the need for formation of a glial scar, which limits expansion of brain damage by isolating the injured tissue from its surroundings and preventing the spread of injurious processes (60). Rapidly expanding astrocyte processes create a wall around the damaged tissue and create time for endogenous repair processes, like neurogenesis, to kick in and repair the surrounding tissue (60). 
Following MSC transplantation, there is also a significant increase in the formation of new neurons from the proliferating progenitors in the damaged hemisphere $(28,29)$. However, there are questions about the ability of recently differentiated neurons to survive long term after an insult. It has been shown in an adult stroke model that only $20 \%$ of the new neurons survive during the first weeks after stroke (62). Newly formed neurons will most likely die when they do not receive appropriate trophic signals or establish functional synaptic connections (63). The latter shows that the "basal" environment or the growth-promoting niche in the brain after ischemic injury is not sufficient for terminal neuronal differentiation, either because of the presence of inhibiting signals and/or because of the lack of instructive cues. For new neurons to survive, the trophic stimulus given by transplanted MSCs may be a crucial factor. Interestingly, MSC transplantation increased expression of synaptophysin, growth-associated protein 43 , and connexin- 43 in the ischemic hemisphere $(29,64)$. Astrocytes express the gap junction protein connexin-43, which plays an important role in intercellular communication. The increased expression of connexin- 43 after MSC treatment is indicative for increased communication between astrocytes and neurons, and this communication contributes to neuronal survival, ion homeostasis, uptake of neurotransmitters, axon myelination, neurogenesis, and synaptogenesis (64).

\section{INFLAMMATION AND MSC-INDUCED REGENERATION OF THE BRAIN}

An important mediator of neurogenesis and other repair processes is inflammation. The inflammatory processes can be detrimental to stimulatory for neurogenesis and other repair processes $(43,65)$.

Several cell types and their activation states influence the inflammatory processes, but microglia are major contributors. Microglia are the resident macrophages of the central nervous system. They are bone marrow-derived monocytes that migrate to the brain during development and differentiate locally into resident microglia $(43,66)$. Upon a stressor or injury, both activation of resident brain microglia and recruitment of monocytes from the periphery contribute to the increase in the number of microglia/macrophages in the injured area $(43,65)$.

Macrophage/microglia heterogeneity and plasticity is great. Microglia can shift their activation state depending on their microenvironment. Under normal conditions, microglia are referred to as "resting," during which they monitor their environment for signals from surrounding cells and have a low expression of protein and surface antigens (67). During brain injury, microglia undergo a rapid shift in their effector program and become activated. Depending on the surrounding conditions, microglia shift their activity state to become "classically activated" M1 microglia or "alternatively activated" M2 microglia $(66,67)$. M1 microglia are a major source of proinflammatory cytokines, oxidative metabolites, and other neurotoxins and thereby contribute to ensuing damage, whereas
M2-activated microglia can be neuroprotective and can promote repair by releasing growth factors like IL10, insulin growth factor- 1 , and transforming growth factor- $\beta$ and modulating the immune response (68). However, knowledge about contribution of these specific subsets in the nervous system is relatively scarce when compared with other tissue macrophages.

In vivo evidence showed that microglial activity can inhibit neurogenesis because there is a negative correlation between the number of activated microglia and the number of surviving new hippocampal neurons (69). Microglia derived from aging mice exhibit a more proinflammatory profile and confer decreased neurogenesis when cultured in vitro together with young NPCs, a phenomenon that may be related to the decrease in neurogenesis during aging (70).

MSC treatment after ischemic brain injury reduces the number of proliferating microglia in the lesioned hemisphere $(28,40)$. The reduced number of microglia after MSC treatment could reflect a reduced number of proinflammatory microglia because MSC transplantation also results in decreased expression of proinflammatory cytokines in the ischemic hemisphere $(29,41)$. By reducing microglial expansion, MSC treatment may reduce inflammation and thus favor formation and integration of new neurons. A recent study using a model for spinal cord injury showed that administration of MSCs induces a shift in microglia activation state from a proinflammatory $\mathrm{M} 1$ state to a repairpermissive M2 state and thereby promoted a regenerative growth response (71).

These M2 microglia could also be responsible for the instructive role microglia can have on neurogenesis. Activated microglia support the migration of NPCs toward the lesion site and stimulate survival of these cells (72). As described above, insulin growth factor-1 is expressed by M2-activated microglia. Activated microglia secreting insulin growth factor- 1 and other factors increase differentiation of neural precursor cells toward neurons when neuronal precursor cells were cultured with microglial-conditioned medium (70).

It is conceivable that MSC transplantation after ischemic brain injury modulates the microenvironment favoring the switch of microglia from a proinflammatory M1 phenotype to a regenerative $\mathrm{M} 2$ phenotype and thereby stimulating regenerative processes.

\section{AXONAL REMODELING AFTER MSC TREATMENT}

The corticospinal tract represents the primary transmission tract for brain-controlled voluntary movement and consists of axons from pyramidal neurons originating in the motor cortex extending to the contralateral spinal cord (73). After an extensive unilateral lesion in the brain, the contralesional hemisphere can contribute to partial recovery of motor function by remodeling of the corticospinal tract $(73,74)$. However, within the damaged hemisphere, axonal remodeling also takes place (75). This remodeling consists of axonal sprouting of new and surviving neurons, neurite extension, synaptogenesis, and 
remyelination $(52,73)$. In neonatal mice with ischemic brain injury, normal axonal routing of the corticospinal tract is disrupted. After injection of the anterograde neuronal tracer biotinylated dextran amine (BDA) into the ipsilesional motor cortex, BDA labeling in the contralesional spinal cord could be determined as a measure of corticospinal tract activity. MSC treatment markedly increased the HI-induced decrease in BDA labeling in the spinal cord (29). Furthermore, BDA labeling in the spinal cord was inversely related to sensorimotor impairment (29).

After neonatal ischemic brain injury, there is a decrease in the amount of white matter (1). Oligodendrocyte progenitors are especially vulnerable to $\mathrm{HI}$ brain injury, which could relate to the characteristic white matter injury in human perinatal brain injury $(76,77)$. Loss of oligodendrocytes leads to demyelination and subsequently axonal conduction will be impaired or lost. In response to MSC transplantation after brain ischemia, there is a large increase in new oligodendrocyte progenitor cells, mature oligodendrocytes, and myelin formation in the ischemic hemisphere $(28,29)$. In vitro MSCs have been shown to promote oligodendroglial fate and to inhibit astroglial fate in neural progenitor cells by factors secreted by the MSCs (78). Neural progenitor cells cultured with factors known to stimulate oligodendrocyte differentiation like insulin growth factor-1, ciliary neurotrophic factor, and nerve growth factor showed that none of the factors tested was as potent as the combination of factors secreted by MSCs (79). We hypothesize that factors secreted by MSCs could contribute to end-stage differentiation of oligodendrocytes, leading to more mature myelin-producing oligodendrocytes and thereby contributing to improved outcome.

Previously, we discussed the beneficial effects of astrocytes and the glial scar on regeneration of the brain. However, the glial scar may also act as a barrier for repair processes because it contains growth inhibitory proteins like myelin-derived proteins and proteoglycans (60). Transplantation of MSCs in adult models for ischemic brain injury has been shown to reduce expression of proteins by astrocytes that are inhibitory to axonal sprouting including Neurocan and Nogo $(80,81)$. Thereby, MSC treatment creates a niche permissive for axonal sprouting, white matter remodeling, and synaptogenesis after ischemic brain injury. Whether this also takes place after neonatal ischemic brain injury remains unknown. However, there are some clues that MSC treatment in the neonatal brain provides an environment promoting axonal sprouting and synaptogenesis as promoting proteins like GAP43, neuropilin-1 and 2, and neuregulin-1 are upregulated in the ischemic hemisphere after MSC treatment (Figure 2) $(29,41)$.

\section{LIMITATIONS AND POTENTIAL ADVERSE EFFECTS}

Until now, no long-term adverse effects have been detected after MSC transplantation to treat ischemic brain injury $(82,83)$. Patients with ischemic stroke and treated with MSCs showed no adverse effects $5 \mathrm{y}$ after treatment with MSCs (82). Also in a rat model, 1-y follow-up did not show any adverse effects (83). Furthermore, there is much evidence that long-term engraftment of MSCs in the brain is limited. However, more research is needed to make firm conclusions about the longterm safety of MSC transplantation.

As with any cell-based therapy, malignant transformation is a potential risk. This risk is high when using embryonic stem cells or induced-pluripotent stem cells. When using adult stem cells the risk is lower, although the risk of malignant transformation increases when MSCs undergo in vitro expansion. However, careful phenol and karyotypic analysis of MSCs pretransplantation should diminish the possibility of transplanting malignant cells. It has also been described by Horn et al. (84) that MSCs may become cytotoxic to hippocampal neurons after oxygen glucose deprivation, which may lead to more damage. Moreover, it is still an open question how MSCs, which only reside for a short period in the brain, can induce long-term recovery. Future research will have to show whether transplanted MSCs redirect or stimulate the autologous stem cell pool, which could lead to sustained functional and anatomical regeneration.

\section{FUTURE PERSPECTIVES}

Transplantation of MSCs is an effective way to repair the damaged brain in an animal for neonatal HI brain injury.

MSC transplantation enhances endogenous cell proliferation and differentiation and results in decreased lesion volume and improved motor function. The administered MSCs do not survive very long in the brain of the recipient, but long enough to stimulate endogenous repair processes. Stimulation of repair by MSCs is mediated via growth factors and is dependent on the bidirectional interplay between the administered MSCs and the ischemic environment in the brain. MSCs can sense the danger in the cerebral environment and will adapt their growth and differentiation factor profile to the demands of the environment, converting it into a repair-promoting environment. The communication between endogenous and exogenous stem cells has significant effects on the behavior of both transplanted and resident stem cells. The potential of meaningful repair of the brain after injury will depend on that bidirectional communication. Manipulation of the expression profiles of growth and differentiation factors in the brain may be crucial for the repair of brain injury in the future. The neonatal brain, which is still developing, probably has a greater potential to regenerate than the adult brain in view of the greater plasticity of the young brain compared with the adult brain. The many processes that are active during development of the brain in the neonate are also needed for repairing injury to the brain. In other words, the neonatal brain is already in a repair-ready state; when injury ensues, the reaction is almost instantaneous and regeneration is more effective than in the adult brain.

\section{REFERENCES}

1. Ferriero DM. Neonatal brain injury. N Engl J Med 2004;351:1985-95.

2. Northington FJ, Chavez-Valdez R, Martin LJ. Neuronal cell death in neonatal hypoxia-ischemia. Ann Neurol 2011;69:743-58.

3. Groenendaal F, Brouwer AJ. Clinical aspects of induced hypothermia in full term neonates with perinatal asphyxia. Early Hum Dev 2009;85:73-6. 
4. Thoresen M. Hypothermia after perinatal asphyxia: selection for treatment and cooling protocol. J Pediatr 2011;158(2 Suppl):e45-9.

5. Okazaki T, Magaki $\mathrm{T}$, Takeda $\mathrm{M}$, et al. Intravenous administration of bone marrow stromal cells increases survivin and Bcl-2 protein expression and improves sensorimotor function following ischemia in rats. Neurosci Lett 2008;430:109-14.

6. Caplan AI, Dennis JE. Mesenchymal stem cells as trophic mediators. J Cell Biochem 2006;98:1076-84.

7. Le Blanc K. Immunomodulatory effects of fetal and adult mesenchymal stem cells. Cytotherapy 2003;5:485-9.

8. Krampera M, Glennie S, Dyson J, et al. Bone marrow mesenchymal stem cells inhibit the response of naive and memory antigen-specific $\mathrm{T}$ cells to their cognate peptide. Blood 2003;101:3722-9.

9. Pimentel-Coelho PM, Mendez-Otero R. Cell therapy for neonatal hypoxicischemic encephalopathy. Stem Cells Dev 2010;19:299-310.

10. Najar M, Raicevic G, Boufker HI, et al. Mesenchymal stromal cells use PGE2 to modulate activation and proliferation of lymphocyte subsets: Combined comparison of adipose tissue, Wharton's Jelly and bone marrow sources. Cell Immunol 2010;264:171-9.

11. Yust-Katz S, Fisher-Shoval Y, Barhum Y, et al. Placental mesenchymal stromal cells induced into neurotrophic factor-producing cells protect neuronal cells from hypoxia and oxidative stress. Cytotherapy 2012;14:45-55.

12. Dos Santos F, Andrade PZ, Boura JS, Abecasis MM, da Silva CL, Cabral JM. Ex vivo expansion of human mesenchymal stem cells: a more effective cell proliferation kinetics and metabolism under hypoxia. J Cell Physiol 2010;223:27-35.

13. Hung SC, Pochampally RR, Hsu SC, et al. Short-term exposure of multipotent stromal cells to low oxygen increases their expression of CX3CR1 and CXCR4 and their engraftment in vivo. PLoS ONE 2007;2:e416.

14. Aggarwal S, Pittenger MF. Human mesenchymal stem cells modulate allogeneic immune cell responses. Blood 2005;105:1815-22.

15. Kebriaei P, Robinson S. Treatment of graft-versus-host-disease with mesenchymal stromal cells. Cytotherapy 2011;13:262-8.

16. Griffin MD, Ritter T, Mahon BP. Immunological aspects of allogeneic mesenchymal stem cell therapies. Hum Gene Ther 2010;21:1641-55.

17. English K, Mahon BP. Allogeneic mesenchymal stem cells: agents of immune modulation. J Cell Biochem 2011;112:1963-8.

18. Le Blanc K, Pittenger M. Mesenchymal stem cells: progress toward promise. Cytotherapy 2005;7:36-45.

19. Isakova IA, Dufour J, Lanclos C, Bruhn J, Phinney DG. Cell-dosedependent increases in circulating levels of immune effector cells in rhesus macaques following intracranial injection of allogeneic MSCs. Exp Hematol 2010;38:957-967.e1.

20. Rossignol J, Boyer C, Thinard R, et al. Mesenchymal stem cells induce a weak immune response in the rat striatum after allo or xenotransplantation. J Cell Mol Med 2009;13(8B):2547-58.

21. Zangi L, Margalit R, Reich-Zeliger S, et al. Direct imaging of immune rejection and memory induction by allogeneic mesenchymal stromal cells. Stem Cells 2009;27:2865-74.

22. Rafei M, Campeau PM, Aguilar-Mahecha A, et al. Mesenchymal stromal cells ameliorate experimental autoimmune encephalomyelitis by inhibiting CD4 Th17 T cells in a CC chemokine ligand 2-dependent manner. J Immunol 2009;182:5994-6002.

23. Lee JK, Jin HK, Bae JS. Bone marrow-derived mesenchymal stem cells reduce brain amyloid-beta deposition and accelerate the activation of microglia in an acutely induced Alzheimer's disease mouse model. Neurosci Lett 2009;450:136-41.

24. Levy YS, Bahat-Stroomza M, Barzilay R, et al. Regenerative effect of neural-induced human mesenchymal stromal cells in rat models of Parkinson's disease. Cytotherapy 2008;10:340-52.

25. van Velthoven CT, Kavelaars A, van Bel F, Heijnen CJ. Regeneration of the ischemic brain by engineered stem cells: fuelling endogenous repair processes. Brain Res Rev 2009;61:1-13.

26. Lee JA, Kim BI, Jo CH, et al. Mesenchymal stem-cell transplantation for hypoxic-ischemic brain injury in neonatal rat model. Pediatr Res 2010;67:42-6.
27. Yasuhara T, Matsukawa N, Yu G, et al. Behavioral and histological characterization of intrahippocampal grafts of human bone marrow-derived multipotent progenitor cells in neonatal rats with hypoxic-ischemic injury. Cell Transplant 2006;15:231-8.

28. van Velthoven CT, Kavelaars A, van Bel F, Heijnen CJ. Mesenchymal stem cell treatment after neonatal hypoxic-ischemic brain injury improves behavioral outcome and induces neuronal and oligodendrocyte regeneration. Brain Behav Immun 2010;24:387-93.

29. van Velthoven CT, Kavelaars A, van Bel F, Heijnen CJ. Repeated mesenchymal stem cell treatment after neonatal hypoxia-ischemia has distinct effects on formation and maturation of new neurons and oligodendrocytes leading to restoration of damage, corticospinal motor tract activity, and sensorimotor function. J Neurosci 2010;30:9603-11.

30. Yasuhara T, Hara K, Maki M, et al. Intravenous grafts recapitulate the neurorestoration afforded by intracerebrally delivered multipotent adult progenitor cells in neonatal hypoxic-ischemic rats. J Cereb Blood Flow Metab 2008;28:1804-10.

31. Danielyan L, Schäfer R, von Ameln-Mayerhofer A, et al. Intranasal delivery of cells to the brain. Eur J Cell Biol 2009;88:315-24.

32. van Velthoven CT, Kavelaars A, van Bel F, Heijnen CJ. Nasal administration of stem cells: a promising novel route to treat neonatal ischemic brain damage. Pediatr Res 2010;68:419-22.

33. Prockop DJ. Repair of tissues by adult stem/progenitor cells (MSCs): controversies, myths, and changing paradigms. Mol Ther 2009;17:939-46.

34. Walczak P, Zhang J, Gilad AA, et al. Dual-modality monitoring of targeted intraarterial delivery of mesenchymal stem cells after transient ischemia. Stroke 2008;39:1569-74.

35. Wang Y, Deng Y, Zhou GQ. SDF-1alpha/CXCR4-mediated migration of systemically transplanted bone marrow stromal cells towards ischemic brain lesion in a rat model. Brain Res 2008;1195:104-12.

36. Rosenkranz K, Kumbruch S, Lebermann K, et al. The chemokine SDF-1/ CXCL12 contributes to the 'homing' of umbilical cord blood cells to a hypoxic-ischemic lesion in the rat brain. J Neurosci Res 2010;88: 1223-33.

37. Shichinohe H, Kuroda S, Yano S, Hida K, Iwasaki Y. Role of SDF-1/CXCR4 system in survival and migration of bone marrow stromal cells after transplantation into mice cerebral infarct. Brain Res 2007;1183:138-47.

38. Mezey E, Chandross KJ, Harta G, Maki RA, McKercher SR. Turning blood into brain: cells bearing neuronal antigens generated in vivo from bone marrow. Science 2000;290:1779-82.

39. Kopen GC, Prockop DJ, Phinney DG. Marrow stromal cells migrate throughout forebrain and cerebellum, and they differentiate into astrocytes after injection into neonatal mouse brains. Proc Natl Acad Sci USA 1999;96:10711-6.

40. Zhang ZG, Chopp M. Neurorestorative therapies for stroke: underlying mechanisms and translation to the clinic. Lancet Neurol 2009;8:491-500.

41. van Velthoven CT, Kavelaars A, van Bel F, Heijnen CJ. Mesenchymal stem cell transplantation changes the gene expression profile of the neonatal ischemic brain. Brain Behav Immun 2011;25:1342-8.

42. Qu R, Li Y, Gao Q, et al. Neurotrophic and growth factor gene expression profiling of mouse bone marrow stromal cells induced by ischemic brain extracts. Neuropathology 2007;27:355-63.

43. Ekdahl CT, Kokaia Z, Lindvall O. Brain inflammation and adult neurogenesis: the dual role of microglia. Neuroscience 2009;158:1021-9.

44. Carlson NG, Wieggel WA, Chen J, Bacchi A, Rogers SW, Gahring LC. Inflammatory cytokines IL-1 alpha, IL-1 beta, IL-6, and TNF-alpha impart neuroprotection to an excitotoxin through distinct pathways. J Immunol 1999;163:3963-8.

45. Loddick SA, Turnbull AV, Rothwell NJ. Cerebral interleukin-6 is neuroprotective during permanent focal cerebral ischemia in the rat. J Cereb Blood Flow Metab 1998; 18:176-9.

46. Mason JL, Suzuki K, Chaplin DD, Matsushima GK. Interleukin-1beta promotes repair of the CNS. J Neurosci 2001;21:7046-52.

47. Gritti A, Frölichsthal-Schoeller P, Galli R, et al. Epidermal and fibroblast growth factors behave as mitogenic regulators for a single multipotent stem cell-like population from the subventricular region of the adult mouse forebrain. J Neurosci 1999;19:3287-97. 
48. Reynolds BA, Weiss S. Generation of neurons and astrocytes from isolated cells of the adult mammalian central nervous system. Science 1992;255:1707-10.

49. Kobayashi T, Ahlenius H, Thored P, Kobayashi R, Kokaia Z, Lindvall O. Intracerebral infusion of glial cell line-derived neurotrophic factor promotes striatal neurogenesis after stroke in adult rats. Stroke 2006;37:2361-7.

50. Shen LH, Li Y, Chopp M. Astrocytic endogenous glial cell derived neurotrophic factor production is enhanced by bone marrow stromal cell transplantation in the ischemic boundary zone after stroke in adult rats. Glia 2010;58:1074-81.

51. Lai K, Kaspar BK, Gage FH, Schaffer DV. Sonic hedgehog regulates adult neural progenitor proliferation in vitro and in vivo. Nat Neurosci $2003 ; 6: 21-7$.

52. Carmichael ST. Cellular and molecular mechanisms of neural repair after stroke: making waves. Ann Neurol 2006;59:735-42.

53. Hagg T. Molecular regulation of adult CNS neurogenesis: an integrated view. Trends Neurosci 2005;28:589-95.

54. Ming GL, Song H. Adult neurogenesis in the mammalian brain: significant answers and significant questions. Neuron 2011;70:687-702.

55. Kurozumi K, Nakamura K, Tamiya T, et al. Mesenchymal stem cells that produce neurotrophic factors reduce ischemic damage in the rat middle cerebral artery occlusion model. Mol Ther 2005;11:96-104.

56. Felling RJ, Snyder MJ, Romanko MJ, et al. Neural stem/progenitor cells participate in the regenerative response to perinatal hypoxia/ischemia. J Neurosci 2006;26:4359-69.

57. Dupret D, Revest JM, Koehl M, et al. Spatial relational memory requires hippocampal adult neurogenesis. PLoS ONE 2008;3:e1959.

58. Raber J, Fan Y, Matsumori Y, et al. Irradiation attenuates neurogenesis and exacerbates ischemia-induced deficits. Ann Neurol 2004;55:381-9.

59. Robel S, Berninger B, Götz M. The stem cell potential of glia: lessons from reactive gliosis. Nat Rev Neurosci 2011;12:88-104.

60. Sofroniew MV. Molecular dissection of reactive astrogliosis and glial scar formation. Trends Neurosci 2009;32:638-47.

61. Pekny M, Nilsson M. Astrocyte activation and reactive gliosis. Glia 2005;50:427-34.

62. Arvidsson A, Collin T, Kirik D, Kokaia Z, Lindvall O. Neuronal replacement from endogenous precursors in the adult brain after stroke. Nat Med 2002;8:963-70.

63. Leker RR, Lasri V, Chernoguz D. Growth factors improve neurogenesis and outcome after focal cerebral ischemia. J Neural Transm 2009;116:1397-402.

64. Zhang C, Li Y, Chen J, et al. Bone marrow stromal cells upregulate expression of bone morphogenetic proteins 2 and 4, gap junction protein connexin-43 and synaptophysin after stroke in rats. Neuroscience 2006;141:687-95

65. Molina-Holgado E, Molina-Holgado F. Mending the broken brain: neuroimmune interactions in neurogenesis. J Neurochem 2010;114:1277-90.

66. Czeh M, Gressens P, Kaindl AM. The yin and yang of microglia. Dev Neurosci 2011;33:199-209.

67. Hanisch UK, Kettenmann H. Microglia: active sensor and versatile effector cells in the normal and pathologic brain. Nat Neurosci 2007;10:1387-94.
68. Schwartz M, Butovsky O, Brück W, Hanisch UK. Microglial phenotype: is the commitment reversible? Trends Neurosci 2006;29:68-74.

69. Yrjänheikki J, Keinänen R, Pellikka M, Hökfelt T, Koistinaho J. Tetracyclines inhibit microglial activation and are neuroprotective in global brain ischemia. Proc Natl Acad Sci USA 1998;95:15769-74.

70. Aarum J, Sandberg K, Haeberlein SL, Persson MA. Migration and differentiation of neural precursor cells can be directed by microglia. Proc Natl Acad Sci USA 2003;100:15983-8.

71. Busch SA, Hamilton JA, Horn KP, et al. Multipotent adult progenitor cells prevent macrophage-mediated axonal dieback and promote regrowth after spinal cord injury. J Neurosci 2011;31:944-53.

72. Thored P, Heldmann U, Gomes-Leal W, et al. Long-term accumulation of microglia with proneurogenic phenotype concomitant with persistent neurogenesis in adult subventricular zone after stroke. Glia 2009;57: 835-49.

73. Dancause N, Barbay S, Frost SB, et al. Extensive cortical rewiring after brain injury. J Neurosci 2005;25:10167-79.

74. Daadi MM, Davis AS, Arac A, et al. Human neural stem cell grafts modify microglial response and enhance axonal sprouting in neonatal hypoxicischemic brain injury. Stroke 2010;41:516-23.

75. Liu Z, Li Y, Zhang ZG, et al. Bone marrow stromal cells enhance inter- and intracortical axonal connections after ischemic stroke in adult rats. J Cereb Blood Flow Metab 2010;30:1288-95.

76. Back SA, Han BH, Luo NL, et al. Selective vulnerability of late oligodendrocyte progenitors to hypoxia-ischemia. J Neurosci 2002;22:455-63.

77. de Vries LS, Groenendaal F. Patterns of neonatal hypoxic-ischaemic brain injury. Neuroradiology 2010;52:555-66.

78. Rivera FJ, Couillard-Despres S, Pedre X, et al. Mesenchymal stem cells instruct oligodendrogenic fate decision on adult neural stem cells. Stem Cells 2006;24:2209-19.

79. Rivera FJ, Kandasamy M, Couillard-Despres S, et al. Oligodendrogenesis of adult neural progenitors: differential effects of ciliary neurotrophic factor and mesenchymal stem cell derived factors. J Neurochem 2008;107:832-43.

80. Li Y, Chen J, Zhang CL, et al. Gliosis and brain remodeling after treatment of stroke in rats with marrow stromal cells. Glia 2005;49: 407-17.

81. Shen LH, Li Y, Gao Q, Savant-Bhonsale S, Chopp M. Down-regulation of neurocan expression in reactive astrocytes promotes axonal regeneration and facilitates the neurorestorative effects of bone marrow stromal cells in the ischemic rat brain. Glia 2008;56:1747-54.

82. Lee JS, Hong JM, Moon GJ, Lee PH, Ahn YH, Bang OY; STARTING collaborators. A long-term follow-up study of intravenous autologous mesenchymal stem cell transplantation in patients with ischemic stroke. Stem Cells 2010;28:1099-106.

83. Shen LH, Li Y, Chen J, et al. One-year follow-up after bone marrow stromal cell treatment in middle-aged female rats with stroke. Stroke 2007;38:2150-6.

84. Horn AP, Frozza RL, Grudzinski PB, et al. Conditioned medium from mesenchymal stem cells induces cell death in organotypic cultures of rat hippocampus and aggravates lesion in a model of oxygen and glucose deprivation. Neurosci Res 2009;63:35-41. 